\title{
Headache, anxiety and depressive disorders: the HADAS study
}

\author{
Ettore Beghi - Gennaro Bussone - Domenico D'Amico • Pietro Cortelli • \\ Sabina Cevoli · Gian Camillo Manzoni - Paola Torelli • Maria Clara Tonini · \\ Giovanni Allais • Roberto De Simone • Florindo D'Onofrio • Sergio Genco • \\ Franca Moschiano $\cdot$ Massimiliano Beghi $\cdot$ Sara Salvi
}

Received: 21 October 2009/Accepted: 26 December 2009/Published online: 27 January 2010

(C) Springer-Verlag 2010

\begin{abstract}
The objective of this paper was to assess prevalence and characteristics of anxiety and depression in migraine without aura and tension-type headache, either isolated or in combination. Although the association between headache and psychiatric disorders is undisputed, patients with migraine and/or tension-type headache have been frequently investigated in different settings and using different tests, which prevents meaningful comparisons. Psychiatric comorbidity was tested through structured interview and the MINI inventory in 158 adults with migraine without aura and in 216 persons with tension-type
\end{abstract}

\section{E. Beghi}

Dipartimento di Neuroscienze,

Istituto "Mario Negri", Milan, Italy

G. Bussone · D. D'Amico

Dipartimento Neuroscienze Cliniche,

Fondazione IRCCS Istituto Neurologico C. Besta, Milan, Italy

P. Cortelli $\cdot$ S. Cevoli

Dipartimento di Scienze Neurologiche,

Università degli Studi di Bologna, Bologna, Italy

G. C. Manzoni $\cdot$ P. Torelli

Centro Cefalee, Sezione di Neurologia, Dipartimento di

Neuroscienze, Università degli Studi di Parma, Parma, Italy

M. C. Tonini

Centro Cefalee Ospedale "G.Salvini",

Garbagnate Milanese (MI), Italy

G. Allais

Centro Cefalee Della Donna,

Dipartimento Discipline Ginecologiche ed Ostetriche,

Università degli studi di Torino, Torino, Italy

R. De Simone

Centro Cefalee e Clinica Neurologica,

Università Federico II, Naples, Italy headache or migraine plus tension-type headache. 49 patients reported psychiatric disorders: migraine 10.9\%, tension-type headache $12.8 \%$, and migraine plus tensiontype headache $21.4 \%$. The MINI detected a depressive episode in 59.9, 67.0, and $69.6 \%$ of cases. Values were $18.4,19.3$, and $18.4 \%$ for anxiety, $12.7,5.5$, and $14.2 \%$, for panic disorder and 2.3, 1.1 and 9.4\% $(p=0.009)$ for obsessive-compulsive disorder. Multivariate analysis showed panic disorder prevailing in migraine compared with the other groups (OR 2.9; 95\% CI 1.2-7.0). The association was higher (OR 6.3; 95\% CI 1.4-28.5) when

F. D’Onofrio

Unità Operativa Complessa di Neurologia, Azienda Ospedaliera "SG Moscati" Avellino, Avellino, Italy

S. Genco

Clinica Neurologica I, Azienda Ospedaliera-Policlinico Bari,

Bari, Italy

F. Moschiano

Unità Operativa Neurologia-Ospedale di Merate (LC),

AO Lecco, Italy

M. Beghi

Clinica Psichiatrica, Università di Milano-Bicocca,

Monza, Italy

S. Salvi

Area Terapeutica, SNC Direzione Medica,

GSK, Verona, Italy

E. Beghi $(\bowtie)$

Istituto di Ricerche Farmacologiche "Mario Negri",

Via G. la Masa 19, 20156 Milan, Italy

e-mail: ettore.beghi@marionegri.it 
migraine (with or without tension-type headache) was compared to pure tension-type headache. This also applied to obsessive-compulsive disorder (OR 4.8; 95\% CI 1.120.9) in migraine plus tension-type headache. Psychopathology of primary headache can reflect shared risk factors, pathophysiologic mechanisms, and disease burden.

Keywords Migraine - Tension-type headache . Depression · Anxiety · Prevalence

\section{Introduction}

The association between headache and psychiatric disorders is undisputed, with depression, bipolar disorders, and anxiety, and somatoform disorders being the commonest complaints [1-3]. Common pathogenic mechanisms between migraine and mood disorders have been implicated, including a decrease of platelet serotonin concentrations, an increase of urinary 5-hydroxytryptamine, and a possible increase of 5-hydroxyindole acetic acid [4]. However, the mechanisms underlying the purported association between migraine and mood disorders are complex, as indicated by the results of prospective studies, which showed a bidirectional influence [5].

Psychiatric comorbidity has been also observed in patients with tension-type headache and may be explained by affective distress, personality disorders, and maladaptive coping [6]. However, patients with migraine and tensiontype headache have been frequently investigated in different settings and using different tests, which prevents meaningful comparisons. For this reason, we decided to undertake an observational study in which patients with migraine could be compared with patients with tension-type headache in terms of prevalence and types of common psychiatric conditions. The main aim of the study was to calculate the prevalence of anxiety disorders and depression, as defined by the DSM-IV diagnostic categories [7] in a naturalistic sample of patients with migraine without aura and in a control group including patients with tension-type headache with or without migraine without aura. Secondary objectives were as follows: (1) to verify the association between anxiety disorders and headache-type (migraine without aura, tension-type headache, migraine plus tensiontype headache); (2) to verify the association between depression and headache type (migraine without aura, tension-type headache, migraine plus tension-type headache).

\section{Materials and methods}

This is a multicenter cross-sectional study done in patients referred to 16 secondary and tertiary headache centers located in Northern and Southern Italy. The criteria for a patient to be enrolled were as follows: age 18 years or more; diagnosis of migraine without aura, and/or tensiontype headache according to the revised international classification of headache disorders [8]; to be a resident of the center catchment area; to be first diagnosed in the participating center; ability to understand Italian language, and to be willing to release a written informed consent. Patients with migraine with aura, chronic migraine, and medicationoveruse headache were excluded. The above inclusion criteria were adopted to identify a well-defined adult population and minimize selection bias.

For each eligible patient, the main demographic features (age, sex, education, occupation, marital status, residency), clinical headache features (disease duration, frequency, duration and severity of attacks, number of days per month with pain, concurrent autonomic symptoms, functional impairment), provoking factors (smoking, alcohol, coffee and tea consumption), coexisting somatic and psychiatric disorders (broad diagnostic categories), and drug treatments were specifically investigated during history taking and recorded in a structured questionnaire.

Each somatic and psychiatric disorder was recorded if deemed clinically relevant by the caring physician. The prevalence of psychiatric disturbances was also assessed by the caring neurologist using the Italian version of the Mini International Neuropsychiatry Interview (MINI) [9]. The MINI is an easy structured diagnostic interview with different sections exploring the principal DSM-IV (Axis I) psychiatric conditions. The ease of use is reflected by the modular characteristics of the interview, which allow the investigator to use the most suitable sections. For the purposes of the present investigation, the following sections were used: (1) major depressive episode; (2) panic disorder; (3) obsessive-compulsive disorder; and (4) generalized anxiety disorder.

The MINI was also used to define the prevalence of depression subtypes (sub-threshold depression, minor depression, secondary depression, and major depressive episodes), anxiety subtypes (secondary or generalized anxiety), and panic and obsessive-compulsive disorder. The presence of a coexistent psychiatric disorder was tested in patients with migraine without aura compared with the other two diagnostic categories. Prior to the start of the study, the local investigators were trained by a psychiatrist to the use of the MINI interview. Training consisted in the organization of a teaching session in the presence of all the local investigators with explanation of the characteristics of the interview and its use in clinical practice. No formal testing of inter-rater agreement was performed.

Where possible, each participating center was asked to enroll the first 45 eligible patients in the following pattern: 15 patients with migraine without aura and 30 patients with 
frequent episodic or chronic and frequent tension-type headache with or without migraine without aura.

A sample size calculation was attempted under the assumption of a $20 \%$ expected prevalence of (major) depression among patients with tension-type headache. Assuming that the prevalence of depression in patients with migraine without aura was about 30\% (10\% absolute difference), a total of 675 patients ( 225 with migraine without aura and 450 with tension-type headache with or without migraine) were to be enrolled with an $80 \%$ power and a $5 \%$ significance level. However, as the recruitment rate was lower than expected, recruitment was stopped when about $50 \%$ of the entire study population was available. With this sample, the study was powered to detect a $35 \%$ difference under the same circumstances.

The statistical analysis plan included descriptive and inferential statistics. Descriptive statistics (means with standard deviation, SD; quartiles; percentages) were used to compare the main demographic and clinical characteristics of the three diagnostic groups (migraine without aura, tension-type headache, migraine plus tension-type headache). Student's $t$ test and analysis of variance (ANOVA) were used as appropriate to compare mean values of continuous variables. Chi-square test was used to compare the prevalence of anxiety and depression in the two main diagnostic groups (migraine without aura; tension-type headache with/without migraine without aura) and in the three diagnostic groups separately. Multivariate analysis was also performed using a binary logistic regression models and a forward stepwise (likehood ratio) procedure. Migraine versus the other headache categories was the response variable in primary analysis. Secondary analyses included migraine plus tension-type headache versus pure tension-type headache and, respectively, migraine and tension-type headache versus the other two groups. Covariates included the four main MINI categories. Data were adjusted for age, gender, marital status (married or other), education (basic or higher), and occupation (employed or unemployed). Statistical significance was set at the $5 \%$ level.

\section{Results}

Patient recruitment started on 18 April 2005 and ended on 13 October 2006.

The study population included 374 patients with migraine without aura (158), tension-type headache (110) and migraine plus tension-type headache (106). The demographic characteristics of the sample are depicted in Table 1. The sample included 291 women and 75 men (gender was unknown in eight cases). Women were largely predominant in the migraine and combined headache groups. The mean (SD) age was 38.4 years (10.7) in patients with migraine without aura, 40.7 years (14.7) in patients with tension-type headache, and 38.5 years (11.9) in patients with both migraine plus tension-type headache. The three groups were fairly similar with reference to education, occupation, and marital status. Residency was outside the center catchment area or unknown in 24 cases (6.4\%). Smoking, alcohol, coffee, and tea consumption was also evenly distributed (data not shown). Compared with tension-type headache, migraine without aura and migraine plus tension-type headache had an earlier age at onset, longer disease duration, lower number of attacks per month, and more severe and persisting attacks with greater functional impairment (Table 2). Severity and duration of attacks peaked in patients with migraine plus tension-type headache. Autonomic symptoms (represented by photophobia, phonophobia, nausea, and to a lesser extent, vomiting) were present in all patients with migraine without aura. One or more provoking factors were reported by 150 patients with migraine without aura $(95.5 \%), 82$ patients with tension-type headache (74.5\%), and 91 patients with migraine plus tension-type headache (88.3\%). Provoking factors had a slightly but significantly different distribution across diagnostic categories (Table 2). One hundred and fifty-three patients with migraine without aura $(96.8 \%)$ were receiving at least one symptomatic treatment as compared with 82 patients with tension-type headache $(77.4 \%)$ and 96 patients with migraine plus tension-type headache $(93.2 \%)$. The corresponding numbers for prophylactic treatment were, respectively, $30(21.3 \%), 27$ (27.0\%), and 39 (39.4\%).

One or more coexisting disorders were present in 66 patients with migraine without aura $(47.8 \%), 50$ patients with tension-type headache (53.2\%), and 59 patients with migraine plus tension-type headache (57.3\%). Psychiatric disorders were the commonest complaints and were identified by history taking in 49 patients (14.6\%; migraine without aura 10.9\%; tension-type headache $12.8 \%$; migraine plus tension-type headache $21.4 \%)(p=0.08)$ (Table 3). Depression was found in $3.6 \%$ of patients with migraine, $8.5 \%$ of patients with tension-type headache, and $10.7 \%$ of patients with migraine plus tension-type headache $(p=0.09)$. The corresponding values for anxiety were $6.5,6.4$, and $14.6 \%(p=0.06)$.

When data deriving from administration of the MINI were analyzed, a depressive episode was recorded in 88 patients with migraine without aura (59.9\%), 71 patients with tension-type headache $(67.0 \%)$, and 71 patients with migraine plus tension-type headache $(69.6 \%)$ (Table 4). When comparing migraine without aura with tensiontype headache with/without migraine, depression subtypes were significantly different $(p=0.03)$. In contrast, the prevalence of anxiety, panic, and obsessive-compulsive 
Table 1 Demographic characteristics of the sample by diagnostic category

\begin{tabular}{|c|c|c|c|c|c|c|c|}
\hline \multirow[t]{2}{*}{ Variable } & \multirow[t]{2}{*}{ Total } & \multicolumn{2}{|c|}{ Migraine without aura } & \multicolumn{2}{|c|}{ Tension-type headache } & \multicolumn{2}{|c|}{ Migraine and tension-type headache } \\
\hline & & $N$ & $\%$ & $N$ & $\%$ & $N$ & $\%$ \\
\hline \multicolumn{8}{|l|}{ Gender* } \\
\hline Men & 75 & 27 & 17.1 & 37 & 34.9 & 11 & 10.8 \\
\hline Women & 291 & 131 & 82.9 & 69 & 65.1 & 91 & 89.2 \\
\hline NS & 8 & - & & 4 & & 4 & \\
\hline \multicolumn{8}{|l|}{$\mathrm{Age}^{* *}$} \\
\hline$<30$ & 91 & 32 & 20.6 & 30 & 27.8 & 29 & 28.2 \\
\hline $30-39$ & 112 & 59 & 38.1 & 24 & 22.2 & 29 & 28.2 \\
\hline $40-49$ & 84 & 38 & 24.5 & 21 & 19.4 & 25 & 24.3 \\
\hline $50-59$ & 52 & 21 & 13.5 & 17 & 15.7 & 14 & 13.6 \\
\hline $60+$ & 27 & 5 & 3.2 & 16 & 14.8 & 6 & 5.8 \\
\hline NS & 8 & 3 & & 2 & & 3 & \\
\hline \multicolumn{8}{|l|}{ Education (year) } \\
\hline$<6$ & 22 & 6 & 3.8 & 11 & 10.1 & 5 & 5.0 \\
\hline $6-8$ & 86 & 34 & 21.7 & 31 & 28.4 & 21 & 20.8 \\
\hline $9-13$ & 194 & 88 & 56.1 & 49 & 45.0 & 57 & 56.4 \\
\hline$>13$ & 61 & 27 & 17.2 & 16 & 14.7 & 18 & 17.8 \\
\hline Other & 4 & 2 & 1.3 & 2 & 1.8 & - & - \\
\hline NS & 7 & 1 & & 1 & & 5 & \\
\hline \multicolumn{8}{|l|}{ Occupation } \\
\hline Employee & 174 & 85 & 54.1 & 37 & 33.6 & 52 & 49.5 \\
\hline Self-employed & 45 & 13 & 8.3 & 18 & 16.4 & 14 & 13.3 \\
\hline Student & 29 & 10 & 6.4 & 12 & 10.9 & 7 & 6.7 \\
\hline Housewife & 60 & 24 & 15.3 & 21 & 19.1 & 15 & 14.3 \\
\hline Pensioner & 32 & 12 & 7.6 & 12 & 10.9 & 8 & 7.6 \\
\hline Unemployed & 32 & 13 & 8.3 & 10 & 9.1 & 9 & 8.6 \\
\hline NS & 2 & 1 & & - & & 1 & \\
\hline \multicolumn{8}{|l|}{ Marital status } \\
\hline Single & 129 & 54 & 34.4 & 40 & 36.4 & 35 & 33.0 \\
\hline Married & 225 & 96 & 61.1 & 64 & 58.2 & 65 & 61.3 \\
\hline Divorced & 12 & 3 & 1.9 & 4 & 3.6 & 5 & 4.7 \\
\hline Widowed & 7 & 4 & 2.5 & 2 & 1.8 & 1 & 0.9 \\
\hline NS & 1 & 1 & & - & & - & \\
\hline
\end{tabular}

NS Not specified

$* p<0.0001$

$* * p<0.01$

disorders was fairly similar. A major depressive episode was the most common subtype (migraine without aura $23.1 \%$; tension-type headache $25.5 \%$; migraine plus tension-type headache $32.4 \%$ ), followed by sub-threshold depression $(21.8,22.6$, and 17.6\%), minor depression (14.3, 10.4, and 14.7\%), and secondary depression (0.7, 8.5 , and $4.9 \%)$. Panic disorder was reported by 20 patients with migraine without aura $(12.7 \%)$, six patients with tension-type headache $(5.5 \%)$, and 15 patients with migraine plus tension-type headache $(14.2 \%)(p=0.09)$. The values for obsessive-compulsive disturbance were 2.3,
1.1 , and $9.4 \%(p=0.01)$. Generalized anxiety was reported by 29 patients with migraine without aura (18.4\%), 21 patients with tension-type headache (19.3\%), and 19 patients with migraine plus tension-type headache $(18.4 \%)$. Anxiety largely predominated in all groups.

Panic disorder was the only psychiatric disturbance found to predominate in patients with migraine without aura when compared with the other headache groups (odds ratio, OR 2.9; 95\% confidence interval, CI 1.2-7.0) (Table 5). The association with panic disorder was even higher when migraine with or without tension-type 
Table 2 Main headache characteristics by diagnostic category

\begin{tabular}{|c|c|c|c|c|c|c|c|}
\hline \multirow[t]{2}{*}{ Variable } & \multirow[t]{2}{*}{ Total } & \multicolumn{2}{|c|}{ Migraine without aura } & \multicolumn{2}{|c|}{ Tension-type headache } & \multicolumn{2}{|c|}{ Migraine and tension-type headache } \\
\hline & & $N$ & $\%$ & $N$ & $\%$ & $N$ & $\%$ \\
\hline \multicolumn{8}{|c|}{ Age at onset (year)* } \\
\hline$<10$ & 29 & 13 & 8.2 & 6 & 5.5 & 10 & 9.5 \\
\hline $10-19$ & 151 & 70 & 44.3 & 29 & 26.4 & 52 & 49.5 \\
\hline $20-29$ & 109 & 46 & 29.1 & 29 & 26.4 & 34 & 32.4 \\
\hline $30-39$ & 45 & 20 & 12.7 & 18 & 16.4 & 7 & 6.7 \\
\hline $40+$ & 39 & 9 & 5.7 & 28 & 25.5 & 2 & 1.9 \\
\hline NS & 1 & - & & - & & 1 & \\
\hline \multicolumn{8}{|c|}{ Disease duration (year)* } \\
\hline$\leq 1$ & 21 & 3 & 1.9 & 17 & 15.7 & 1 & 1.0 \\
\hline $2-4$ & 47 & 13 & 8.4 & 20 & 18.5 & 14 & 13.7 \\
\hline $5-9$ & 53 & 22 & 14.2 & 22 & 20.4 & 9 & 8.8 \\
\hline $10-19$ & 99 & 47 & 30.3 & 24 & 22.2 & 28 & 27.5 \\
\hline $20-29$ & 83 & 44 & 28.4 & 12 & 11.1 & 27 & 26.5 \\
\hline$\geq 30$ & 62 & 26 & 16.8 & 13 & 12.0 & 23 & 22.5 \\
\hline NS & 9 & 3 & & 2 & & 4 & \\
\hline \multicolumn{8}{|c|}{ Number of attacks/month* } \\
\hline $1-2$ & 89 & 39 & 25.0 & 7 & 6.9 & 43 & 41.3 \\
\hline $3-4$ & 120 & 72 & 46.2 & 16 & 15.8 & 32 & 30.8 \\
\hline$>4$ & 152 & 45 & 28.8 & 78 & 77.2 & 29 & 27.9 \\
\hline NS & 13 & 2 & & 9 & & 2 & \\
\hline \multicolumn{8}{|c|}{ Duration of attacks (h)** } \\
\hline$<3$ & 10 & 6 & 3.8 & 4 & 3.7 & - & - \\
\hline $3-4$ & 34 & 17 & 10.9 & 12 & 11.1 & 5 & 4.9 \\
\hline $5-24$ & 184 & 77 & 49.4 & 59 & 54.6 & 48 & 47.1 \\
\hline $25-72$ & 123 & 54 & 34.6 & 24 & 22.2 & 45 & 44.1 \\
\hline$>72$ & 15 & 2 & 1.3 & 9 & 8.3 & 4 & 3.9 \\
\hline NS & 8 & 2 & & 2 & & 4 & \\
\hline \multicolumn{8}{|c|}{ Severity of attacks* } \\
\hline Mild & 42 & 1 & 0.7 & 41 & 38.7 & - & - \\
\hline Moderate & 108 & 40 & 26.3 & 51 & 48.1 & 17 & 16.7 \\
\hline Severe & 186 & 101 & 66.4 & 12 & 11.3 & 73 & 71.6 \\
\hline Very severe & 24 & 10 & 6.6 & 2 & 1.9 & 12 & 11.8 \\
\hline NS & 14 & 6 & & 4 & & 4 & \\
\hline \multicolumn{8}{|c|}{ Autonomic symptoms ${ }^{\mathrm{a}}$} \\
\hline Photophobia & 214 & 131 & 83.4 & NS & NS & 83 & 81.4 \\
\hline Phonophobia & 206 & 129 & 82.2 & NS & NS & 77 & 75.5 \\
\hline Nausea & 220 & 133 & 84.7 & NS & NS & 87 & 85.3 \\
\hline Vomiting & 91 & 61 & 38.9 & NS & NS & 30 & 29.4 \\
\hline Other & 48 & 32 & 20.4 & NS & NS & 16 & 15.7 \\
\hline \multicolumn{8}{|c|}{ Provoking factors $* * * a$} \\
\hline Alimentary & 20 & 10 & 6.4 & 1 & 0.9 & 9 & 8.7 \\
\hline Emotions & 77 & 32 & 20.4 & 32 & 29.1 & 13 & 12.6 \\
\hline Stress & 212 & 91 & 58.0 & 63 & 57.3 & 58 & 56.3 \\
\hline Noise & 20 & 8 & 5.1 & 6 & 5.5 & 6 & 5.8 \\
\hline Other & 111 & 58 & 36.9 & 26 & 23.6 & 27 & 26.2 \\
\hline \multicolumn{8}{|c|}{ Functional disability* } \\
\hline None & 16 & 4 & 2.5 & 12 & 10.9 & - & - \\
\hline
\end{tabular}


Table 2 continued

\begin{tabular}{|c|c|c|c|c|c|c|c|}
\hline \multirow[t]{2}{*}{ Variable } & \multirow[t]{2}{*}{ Total } & \multicolumn{2}{|c|}{ Migraine without aura } & \multicolumn{2}{|c|}{ Tension-type headache } & \multicolumn{2}{|c|}{ Migraine and tension-type headache } \\
\hline & & $N$ & $\%$ & $N$ & $\%$ & $N$ & $\%$ \\
\hline Mild & 93 & 12 & 7.6 & 72 & 65.5 & 9 & 8.7 \\
\hline Moderate & 160 & 80 & 51.0 & 22 & 20.0 & 58 & 55.8 \\
\hline Severe & 102 & 61 & 38.9 & 4 & 3.6 & 37 & 35.6 \\
\hline NS & 3 & 1 & & - & & 2 & \\
\hline
\end{tabular}

NS Not specified

$* p<0.0001$

$* * p<0.005$

$* * * p<0.05$

${ }^{a}$ Several patients had two or more autonomic and/or provoking factors

Table 3 Concomitant clinical conditions by diagnostic category

\begin{tabular}{|c|c|c|c|c|c|c|c|}
\hline \multirow[t]{2}{*}{ Variable } & \multirow[t]{2}{*}{ Total } & \multicolumn{2}{|c|}{ Migraine without aura } & \multicolumn{2}{|c|}{ Tension-type headache } & \multicolumn{2}{|c|}{ Migraine and tension-type headache } \\
\hline & & $N$ & $\%$ & $N$ & $\%$ & $N$ & $\%$ \\
\hline Psychiatric & 49 & 15 & 10.9 & 12 & 12.8 & 22 & 21.4 \\
\hline Allergic & 47 & 16 & 11.6 & 11 & 11.7 & 20 & 19.4 \\
\hline Gastrointestinal & 43 & 19 & 13.8 & 9 & 9.6 & 15 & 14.6 \\
\hline Cardiovascular & 34 & 14 & 10.1 & 12 & 12.8 & 8 & 7.8 \\
\hline Endocrine & 34 & 13 & 9.4 & 11 & 11.7 & 10 & 9.7 \\
\hline Skeletal & 25 & 12 & 8.7 & 6 & 6.4 & 7 & 6.8 \\
\hline ENT & 14 & 8 & 5.8 & 3 & 3.2 & 3 & 2.9 \\
\hline Metabolic & 9 & 5 & 3.6 & 3 & 3.2 & 1 & 1.0 \\
\hline Neurological & 8 & 4 & 2.9 & 1 & 1.1 & 3 & 2.9 \\
\hline Respiratory & 7 & 1 & 0.7 & 1 & 1.1 & 5 & 4.9 \\
\hline Renal & 6 & 2 & 1.4 & 1 & 1.1 & 3 & 2.9 \\
\hline Other & 7 & 2 & 1.4 & 2 & 2.1 & 3 & 2.9 \\
\hline
\end{tabular}

ENT Ear, nose, and throat

headache was compared with pure tension-type headache (OR 6.3; 95\% CI 1.4-28.5). In contrast, obsessive-compulsive disorder was the only independent predictor in patients with migraine plus tension-type headache (OR 4.8; 95\% CI 1.1-20.9 vs. those with migraine and tension-type headache separately).

\section{Discussion}

Our study showed that psychiatric disturbances, as reported by the caring physician, are the commonest comorbid complaints in patients with migraine without aura and/or tension-type headache, with depression, anxiety, panic and obsessive-compulsive disorders in decreasing order, and differences across diagnostic groups when these disorders are screened using a standardized psychiatric inventory.
These are not unexpected findings because several studies done in clinical and community-based settings have reported an association between migraine and a number of specific psychiatric disorders [1, 2]. Active screening here was followed by an even higher prevalence of affective and anxiety disorders. Major depression was reported in up to one-third of our cases, in keeping with Breslau and coworkers [10] who found major depression in $40.7 \%$ of persons with migraine and $35.8 \%$ of those with other severe headaches.

Migraine without aura, tension-type headache, and migraine associated with tension-type headache showed significant differences in the rate of occurrence of depression subtypes, the latter group being at higher risk. Duration and severity of attacks peaked in patients with both migraine and tension-type headache suggesting that this diagnostic category, despite being similarly disabling, has a 
Table 4 Selected psychiatric disorders, as recorded by the MINI interview, by diagnostic category

\begin{tabular}{|c|c|c|c|c|c|c|c|}
\hline \multirow[t]{2}{*}{ Psychiatric disorder } & \multirow[t]{2}{*}{ Total } & \multicolumn{2}{|c|}{ Migraine without aura } & \multicolumn{2}{|c|}{ Tension-type headache } & \multicolumn{2}{|c|}{ Migraine and tension-type headache } \\
\hline & & $N$ & $\%$ & $N$ & $\%$ & $N$ & $\%$ \\
\hline \multicolumn{8}{|l|}{ Depressive episode } \\
\hline No & 125 & 59 & 40.1 & 35 & 33.0 & 31 & 30.4 \\
\hline Sub-threshold & 74 & 32 & 21.8 & 24 & 22.6 & 18 & 17.6 \\
\hline Minor & 47 & 21 & 14.3 & 11 & 10.4 & 15 & 14.7 \\
\hline Secondary & 15 & 1 & 0.7 & 9 & 8.5 & 5 & 4.9 \\
\hline Major & 94 & 34 & 23.1 & 27 & 25.5 & 33 & 32.4 \\
\hline NS & 19 & 11 & - & 4 & - & 4 & - \\
\hline \multicolumn{8}{|l|}{ Major depressive episode } \\
\hline Present & 23 & 8 & 28.6 & 5 & 26.3 & 10 & 35.7 \\
\hline Past & 52 & 20 & 71.4 & 14 & 73.7 & 18 & 64.3 \\
\hline NS & 19 & 6 & - & 8 & - & 5 & - \\
\hline \multicolumn{8}{|l|}{ Panic disorder } \\
\hline No & 333 & 138 & 87.3 & 104 & 94.5 & 91 & 85.8 \\
\hline Lifetime panic attacks & 1 & - & - & 1 & 0.9 & - & - \\
\hline Present panic disorder & 10 & 3 & 1.9 & 2 & 1.8 & 5 & 4.7 \\
\hline Lifetime panic disorder & 30 & 17 & 10.8 & 3 & 2.7 & 10 & 9.4 \\
\hline \multicolumn{8}{|c|}{ Obsessive-compulsive disorder* } \\
\hline No & 289 & 125 & 97.7 & 87 & 98.9 & 77 & 90.6 \\
\hline Present & 7 & 2 & 1.6 & - & - & 5 & 5.9 \\
\hline Past & 5 & 1 & 0.8 & 1 & 1.1 & 3 & 3.5 \\
\hline NS & 73 & 30 & & 22 & & 21 & \\
\hline \multicolumn{8}{|l|}{ Anxiety } \\
\hline No & 301 & 129 & 81.6 & 88 & 80.7 & 84 & 81.6 \\
\hline Secondary & 11 & 6 & 3.8 & 4 & 3.7 & 1 & 1.0 \\
\hline Generalised & 57 & 23 & 14.6 & 16 & 14.7 & 18 & 17.5 \\
\hline Undetermined & 1 & - & - & 1 & 0.9 & - & - \\
\hline NS & 4 & - & - & 1 & - & 3 & - \\
\hline \multicolumn{8}{|l|}{ Generalized anxiety } \\
\hline Present & 24 & 8 & 38.1 & 6 & 54.5 & 10 & 71.4 \\
\hline Past & 21 & 13 & 61.9 & 5 & 45.5 & 3 & 21.4 \\
\hline Past and present & 1 & - & - & - & - & 1 & 7.1 \\
\hline NS & 11 & 2 & - & 5 & - & 4 & - \\
\hline
\end{tabular}

NS Not specified

$* p<0.05$

major impact on patient's mood. This finding is in line with others, who observed higher levels of anxiety, depression, phobia, obsessive-compulsive symptoms, and emotional liability in patients with both tension-type and migraine attacks [11]. In addition, symptoms associated with anxiety and depression are more common in patients with chronic migraine than in those with episodic migraine and tensiontype headache $[12,13]$. This suggests that psychopathology of primary headache can be a reflection of the overall burden of the disease rather than a hallmark of a specific headache category.
The most interesting result of this study is the significant correlation found between migraine and panic disorders. A correlation between migraine and panic disorder has been frequently reported [14-19] with a bidirectional association [20]. Shared environmental or genetic risk factors might be involved. Pathophysiologic models of migraine and panic disorder point out to specific neurotransmitters, including the serotonin system [21-23]. A second interesting observation is the correlation between migraine plus tensiontype headache and obsessive-compulsive disorders. Our results are in line with the few studies $[12,24]$ on this topic, 
Table 5 Results of multivariate analysis

\begin{tabular}{|c|c|c|}
\hline \multicolumn{3}{|c|}{ A. Migraine/with/without tension-type headache versus tension-type headache } \\
\hline Independent variable ${ }^{\mathrm{a}}$ & Odds ratio & $95 \%$ confidence interval \\
\hline Panic disorder & 2.9 & $1.2-7.0$ \\
\hline \multicolumn{3}{|c|}{ B. Migraine versus tension-type with/without migraine } \\
\hline \multicolumn{3}{|l|}{ Independent variables ${ }^{\mathrm{b}}$} \\
\hline Panic disorder & 6.3 & $1.4-28.5$ \\
\hline Poor education & 0.5 & $0.3-0.9$ \\
\hline Female gender & 3.6 & $1.8-7.0$ \\
\hline \multicolumn{3}{|c|}{ C. Migraine/tension-type headache versus migraine and tension-type headache } \\
\hline \multicolumn{3}{|l|}{ Independent variables $^{c}$} \\
\hline Obsessive-compulsive disorder & 4.8 & $1.1-21.0$ \\
\hline Female gender & 2.6 & $1.1-6.1$ \\
\hline
\end{tabular}

which found a higher prevalence of obsessive-compulsive disorders in patients with both headache types when compared with migraine and tension headache. There are several possible explanations for these findings. Patients with obsessive-compulsive disorders usually are very precise in the description of their symptoms, pay attention to minor details, and are not able to discriminate between major and minor details (in this case the main and the minor features of their headache). Moreover, chronic headache sufferers show a significant impairment in their ability to control obsessive-compulsive symptoms and there might be an exacerbation of a pre-morbid condition.

In this study, major depression had a significantly higher prevalence among patients with migraine plus tension-type headache, while there were no differences between migraine and tension type headache, and depression was not found to be associated with migraine in multivariate analysis. Our data are at variance with several other clinicand community-based surveys [1, 2, 19]. However, the association has been reported to be higher in patients with migraine with aura than in those with migraine without aura $[11,25,26]$ and has been also observed for severe headache [11]. Merikangas et al. [25] pointed out the greater severity of migraine with aura as a possible explanation of this discrepancy. This latter finding suggests that illness severity, more than a definite pathophysiological mechanism, could be involved in the development of a depressive disorder in patients with headache. In keeping with this, here in the migraine and tension type headache groups less than $30 \%$ of the patients with a major depressive episode reported a current episode compared with more than $35 \%$ of those with both migraine and tensiontype headache combined form. Although weak, our results are in line with the assumption of Hung [27] that a depressive episode may worsen their headache. A previous study [28] found a significant correlation between depression and migraine; however, when considered simple (without head trauma or "transformed" headache), the severity of depressive symptoms is similar to that of tension type headache. In the same way, affective disorders do not seem to predict incident migraine without aura headaches, in line with previous studies [29] and in contrast with others [30].

This study has several limitations. First of all, given some practical constraints (i.e. the limited number of participating centers), a number of patients lower than expected could be enrolled, thus decreasing the power of the study and limiting our analysis. However, based on the prevalence of major depression in patients with migraine without aura $(23.1 \%)$ and in those with tension-type headache $(25.5 \%)$, a significantly greater number of patients would be needed in order for this difference to achieve statistical significance. Second, our population was represented by patients referred to secondary and tertiary centers, who tend to present most commonly severe headache varieties. This may lend support to the concept that in the general population even lower differences could be expected between migraine without aura and pure tension-type headache for psychiatric comorbidity other than panic disorder. In this context, the difference between pure headache types and migraine plus tension-type headache may be less striking. Third, we excluded patients with migraine with aura, chronic migraine, and medication overuse headache. This may have further selected the study population introducing other sources of bias. Fourth, we did not investigate bipolar and somatoform disorders, which were outside the scopes of our survey. This limits the spectrum of the psychiatric disorders possibly 
associated with headache. Fifth, the psychiatric diagnoses were not confirmed by a formal psychiatric evaluation. This may explain at least in part the discrepancy between the prevalence of anxiety and depression as reported during the history taking and the results of the MINI interview. Sixth, in the absence of a control group (i.e. patients without headache from the general population), an exact estimate of the psychiatric risk attributable to migraine and tension-type headache cannot be calculated. Seventh, our sample excludes migraine with aura that is supposed to be the form with the strongest psychiatric comorbidity [11, 25, 26]. Eighth, concerning the obsessive compulsive disorders, a high percentage of data is missing $(19.5 \%)$ and the total number of episodes is very low (seven present and five past episodes). Ninth, data on phobia, one of the most comorbid disorders in patients with headache [11], were not obtained here. Last, several subjects in this study received prophylactic treatment, which might have affected patients' psychopathology.

\section{Conclusion}

Even with the above limitations, the study provides consistent findings in support of a correlation between panic disorder and migraine without aura, which is stronger when migraine and tension-type headaches are both present, and between obsessive-compulsive disorder and migraine with tension-type headache. Shared risk factors and pathophysiologic mechanisms as well as the overall severity of the disease may explain this selective comorbidity.

Acknowledgments The study was performed by ANIRCEF with an educational grant by GSK.

\section{Conflict of interest None.}

\section{References}

1. Pompili M, Di Cosimo D, Innamorati M, Lester D, Tatarelli R, Martelletti P (2009) Psychiatric comorbidity in patients with chronic daily headache and migraine: a selective overview including personality traits and suicide risk. J Headache Pain 10:283-290

2. Hamelsky SW, Lipton RB (2006) Psychiatric comorbidity of migraine. Headache 46:1327-1333

3. Wang SJ, Juang KD (2002) Psychiatric comorbidity of chronic daily headache: impact, treatment, outcome, and future studies. Curr Pain Headache Rep 6:505-510

4. Ferrari MD, Odink J, Tapparelli C, Van Kempen GM, Pennings EJ, Bruyn GW (1989) Serotonin metabolism in migraine. Neurology 39:1239-1242

5. Shechter AL, Lipton RB, Silberstein SD (2001) Migraine comorbidity. In: Silberstein SD, Lipton RB, Dalessio DJ (eds) Wolff's headache and other head pai, 7th edn. University Press, Oxford, pp 108-118
6. Heckman BD, Holroyd KA (2006) Tension-type headache and psychiatric comorbidity. Curr Pain Headache Rep 10:439-447

7. American Psychiatric Association <http://www.psych.org/> (2000) Diagnostic and statistical manual of mental disorders, 4th edn. Washington DC, Text Revision

8. Headache classification subcommittee of the international headache society (2004) The international classification of headache disorders: 2nd edn. Cephalalgia 24(Suppl 1):9-160

9. Sheehan DV, Lecrubier Y, Sheehan KH et al (1998) The MiniInternational Neuropsychiatric Interview (M.I.N.I.): the development and validation of a structured diagnostic psychiatric interview for DSM-IV and ICD-10. J Clin Psychiatry 59(Suppl 20):22-33

10. Breslau N, Davis GC, Andreski P (1991) Migraine, psychiatric disorders and suicide attempts. An epidemiological study of young adults. Psychiatry Res 37:11-23

11. Breslau N, Schultz LR, Stewart WF, Lipton RB, Lucia VC, Welch KM (2000) Headache and major depression: Is the association specific to migraine? Neurology 54:308-313

12. Perozzo P, Savi L, Castelli L et al (2005) Anger and emotional distress in patients with migraine and tension-type headache. J Headache Pain 6:392-399

13. Mongini F, Rota E, Deregibus A et al (2006) Accompanying symptoms and psychiatric comorbidity in migraine and tensiontype headache patients. J Psychosom Res 61:447-451

14. Breslau N, Davis GC (1993) Migraine, physical health and psychiatric disorders: a prospective epidemiologic study in young adults. J Psychiatr Res 27:211-221

15. Stewart WF, Linet MS, Celentano DD (1989) Migraine headaches and panic attacks. Psychosom Med 51:559-569

16. Stewart WF, Shecther A, Liberman J (1992) Physician consultation for headache pain and history of panic: Results from a population-based study. Am J Med 92:35S-40S

17. Stewart WF, Breslau N, Keck PE Jr (1994) Comorbidity of migraine and panic disorder. Neurology 44:23-27

18. Marazziti D, Toni C, Pedri S et al (1999) Prevalence of headache syndromes in panic disorder. Int Clin Psychopharmacol 14:247251

19. Jette N, Patten S, Williams J, Becker W, Wiebe S (2008) Comorbidity of migraine and psychiatric disorders. A national population-based study. Headache 48:501-516

20. Breslau N, Schultz LR, Stewart WF, Lipton R, Welch KMA (2001) Headache types and panic disorder. Directionality and specificity. Neurology 56:350-354

21. Lipton RB, Silberstein SD (1994) Why study the comorbidity of migraine? Neurology 44:S4-S5

22. Bell CJ, Nutt DJ (1998) Serotonin and panic. Br J Psychiatry 172:465-471

23. Loi V, Lai M, Pisano MR, Del Zompo M (1996) Sumatriptan and panic-like symptoms. Am J Psychiatr 153:1505

24. Arena J, Blanchard EB, Andrasik F, Applebaum K (1986) Obsessions and compulsions in three kinds of headache sufferers: analysis of the maudsley questionnaire. Behav Res Ther 2:127-132

25. Merikangas KR, Merikangas JR, Angst J (1993) Headache syndromes and psychiatric disorders: association and familial transmission. J Psychiatr Res 27:197-210

26. Oedegaard KJ, Neckelmann D, Mykletun A et al (2006) Migraine with and without aura: association with depression and anxiety disorder in a population-based study. The HUNT study. Cephalalgia $26: 1-6$

27. Hung CI, Liu CY, Juang YY, Wang SJ (2006) The impact of migraine on patients with major depressive disorders. Headache 46:469-477

28. Jelinski SE, Magnusson JE, Becker WJ, CHORD Study Group (2007) Factors associated with depression in patients referred to headache specialists. Neurology 68:489-495 
29. Swartz KL, Pratt LA, Armenian HK, Lee LC, Eaton WW (2000) Mental disorders and the incidence of migraine headaches in a community. Arch Gen Psychiatr 57:945-950
30. Breslau N, Lipton RB, Stewart WF, Schultz LR, Welch KM (2003) Comorbidity of migraine and depression. Investigating potential etiology and prognosis. Neurology 60:1308-1312 